\title{
Transitions to three-dimensional flows in a cylinder driven by oscillations of the sidewall
}

\author{
C. PANADES $S^{1}$, F. MARQUES ${ }^{1}$ AND J. M. LOPEZ $Z^{2} \dagger$ \\ ${ }^{1}$ Departament de Física Aplicada, Universitat Politècnica de Catalunya, Barcelona 08034, Spain \\ ${ }^{2}$ School of Mathematical and Statistical Sciences, Arizona State University, Tempe, AZ 85287, USA
}

(Received 3 November 2010; revised 15 February 2011; accepted 9 May 2011;

first published online 24 June 2011)

The transition from two-dimensional to three-dimensional flows in a finite circular cylinder driven by an axially oscillating sidewall is explored in detail. The complete symmetry group of this flow, including a spatio-temporal symmetry related to the oscillating sidewall, is $Z_{2} \times O(2)$. Previous studies in flows with the same symmetries, such as symmetric bluff-body wakes and periodically forced rectangular cavities, were unable to obtain the theoretically predicted bifurcation to modulated travelling waves. In the simpler cylindrical geometry, where the azimuthal direction is physically periodic, we have found these predicted modulated travelling waves as stable fully saturated nonlinear solutions for the first time. A careful analysis of the base states and their linear stability identifies different parameter regimes where three-dimensional states are either synchronous with the forcing or quasi-periodic, corresponding to different symmetry-breaking processes. These results are in good agreement with theoretical predictions and previous results in similar flows. These different regimes are separated by three codimension-two bifurcation points that are yet to be fully analysed theoretically. Finally, the saturated nonlinear states and their properties in different parameter regimes are analysed.

Key words: bifurcation, nonlinear instability, parametric instability

\section{Introduction}

When a system is invariant under the action of a group of symmetries, there can be far-reaching consequences on its bifurcations. When the symmetries are purely spatial in nature (e.g. reflections, translations and rotations), these consequences have been extensively studied (e.g. see Golubitsky \& Schaeffer 1985; Golubitsky, Stewart \& Schaeffer 1988; Crawford \& Knobloch 1991; Cross \& Hohenberg 1993; Chossat \& Iooss 1994; Iooss \& Adelmeyer 1998; Chossat \& Lauterbach 2000; Golubitsky \& Stewart 2002; Hoyle 2006). The system may also be invariant to the action of spatio-temporal symmetries. These are spatial symmetries composed with temporal evolution. A classic example is the two-dimensional Kármán vortex street form of the wake of a circular cylinder. Other common cases are periodically forced flows.

The transition from two-dimensional to three-dimensional flow is of fundamental interest in fluid dynamics. Two-dimensional flows, like the Kármán vortex street and other bluff-body wakes, are invariant in the spanwise direction to both translations ( $S O(2)$ symmetry group) and reflections $\left(Z_{2}\right.$ symmetry group), the combination 
generating the $O(2)$ symmetry group. We are interested in the transition from twodimensional to three-dimensional flow when the two-dimensional problem has a spatio-temporal symmetry of $Z_{2}$ type, as is the case for the wake of a circular cylinder in the streamwise direction (Barkley, Tuckerman \& Golubitsky 2000; Blackburn \& Lopez 2003a; Blackburn, Marques \& Lopez 2005). Another flow with the same spatio-temporal symmetries as the periodically shedding wake is that in a periodically driven rectangular cavity of infinite spanwise extent (Marques, Lopez \& Blackburn 2004), which has been studied in Lopez \& Hirsa (2001), Vogel, Hirsa \& Lopez (2003) and Blackburn \& Lopez (2003b).

The complete symmetry group of these flows is $Z_{2} \times O(2)$. The implications of $O(2)$ symmetry in fluid systems have been studied extensively, both when the instability breaking $O(2)$ symmetry (i.e. transition from two-dimensional to three-dimensional) is due to a pair of real eigenvalues becoming positive (steady bifurcation) as well as when it is due to two pairs of complex-conjugate eigenvalues gaining positive real part, leading to time-periodic flow (e.g. see the references cited above); the multiplicity two of the eigenvalues being due to the $O(2)$ symmetry. The types of symmetry-breaking bifurcations to three-dimensional flow, that a two-dimensional flow with a spacetime symmetry can experience, are completely determined by the symmetry group of the system, and not by the particulars of the physical mechanisms responsible for the bifurcation, and have been analysed in detail in Marques et al. (2004). The equivariant branching lemma (see, for example Golubitsky et al. 1988; Chossat \& Lauterbach 2000) formalizes the notion that the symmetries of the system govern the types of possible bifurcations that may occur, as well as the symmetry properties of the bifurcating solutions themselves. The main results obtained for systems with $Z_{2} \times O(2)$ spatio-temporal symmetry are that there are two types of bifurcations, one synchronous with the forcing and the other resulting in quasi-periodic flows. Both types come in two different flavours, depending on the symmetries of the bifurcated solutions. There are two synchronous modes, $\mathrm{A}$ and $\mathrm{B}$, that break or preserve the space-time symmetry $Z_{2}$, respectively. The quasi-periodic solutions have the form of modulated travelling waves or modulated standing waves in the spanwise direction; they differ in their symmetry properties: the travelling waves preserve a space-time symmetry, while the standing wave preserves a purely spatial reflection symmetry.

In the examples of flows with $Z_{2} \times O(2)$ symmetry group described above, the $O(2)$ invariance is only an idealization of the corresponding experimental flow due to the finite extent of the spanwise direction. The typical result is that the travelling waves predicted by the theory do not travel, due to endwall effects (Leung et al. 2005). Cylindrical geometries are very useful in the sense that the azimuthal direction is physically periodic and have the $O(2)$ symmetry group exactly fulfilled. This fact has been explored in Blackburn \& Lopez (2011) in a driven annular geometry, but unfortunately, the modulated travelling wave modes that are predicted from the Floquet analysis do not saturate nonlinearly to pure modes but are always mixed with contributions from the synchronous A mode. In the present paper, we explore a simpler setting, a finite circular cylinder with an axially oscillating sidewall. The base state is also $Z_{2} \times O(2)$ invariant as in the other flow examples, and we have indeed found nonlinearly saturated pure modulated travelling waves for the first time in a physically realizable flow. In this cylindrical setting, as the travelling waves move in the azimuthal direction, i.e. the pattern rotates around the cylinder axis, they will be termed rotating (or modulated rotating) waves.

The paper is organized as follows: in $\S 2$, the formulation of the problem and numerical methods used are presented; in $\S 3$, the base state of the system is computed, 


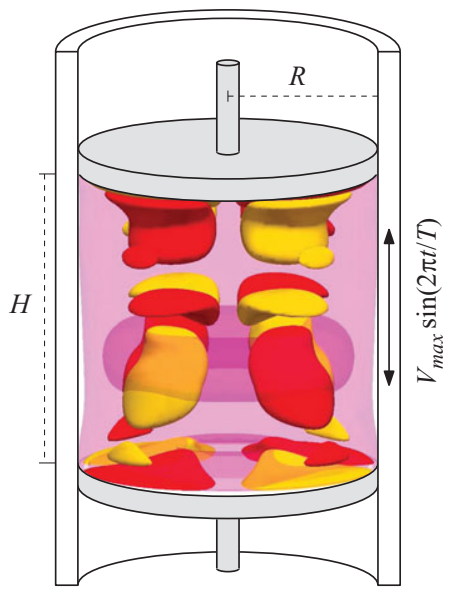

FIGURE 1. (Colour online available at journals.cambridge.org/FLM) Schematic of the apparatus and a snapshot of a synchronous bifurcated state. The translucent isosurfaces (of azimuthal vorticity) show the axisymmetric roller generated by the sidewall oscillation, and solid isosurfaces (of radial vorticity) show the braid structures associated with three-dimensional instabilities.

and its changes when parameters are varied are discussed; in $\S 4$, the linear stability of the basic flow is studied, and compared with similar flows; in $\S 5$, the three-dimensional structure and symmetries of the different unstable modes found are analysed in detail; and finally, in $\S 6$, the main results are summarized and open problems and future directions of study are discussed.

\section{Governing equations and numerical methods}

Consider a Newtonian fluid of kinematic viscosity $v$ confined in a finite cylinder of radius $R$ and height $H$, whose sidewall oscillates harmonically in the axial direction, with period $T$ and maximum axial velocity $V_{\max }$, while the top and bottom lids remain at rest, as shown schematically in figure 1 . The system is non-dimensionalized taking $R$ as the length scale, and the viscous time $R^{2} / \nu$ as the time scale. There are three non-dimensional parameters in this problem:

$$
\begin{array}{ll}
\text { Aspect ratio } & \Gamma=H / R, \\
\text { Reynolds number } & R e=V_{\max } R / \nu, \\
\text { Stokes number } & S t=R^{2} / \nu T .
\end{array}
$$

The aspect ratio defines the geometry of the problem, while Re and St are nondimensional measures of the amplitude and frequency of the forcing; the inverse of the Stokes number is precisely the non-dimensional period of the oscillations, $\tau=1 / S t$. In the current study, the aspect ratio is fixed at $\Gamma=2$. The non-dimensional Navier-Stokes equations governing the flow are

$$
\begin{aligned}
\left(\frac{\partial}{\partial t}+\boldsymbol{u} \cdot \nabla\right) \boldsymbol{u} & =-\nabla p+\nabla^{2} \boldsymbol{u}, \\
\nabla \cdot \boldsymbol{u} & =0,
\end{aligned}
$$


where $\boldsymbol{u}=(u, v, w)$ is the velocity field in cylindrical coordinates $(r, \theta, z) \in$ $[0,1] \times[0,2 \pi] \times[-\Gamma / 2, \Gamma / 2]$, and $p$ is the kinematic pressure. The vorticity associated to the velocity field is $\nabla \times \boldsymbol{u}=(\xi, \eta, \zeta)$. No-slip velocity boundary conditions are used on all walls. The velocity is zero on stationary top and bottom endwalls, and the $z$-component of velocity at the sidewall oscillates periodically in time

$$
\begin{aligned}
\boldsymbol{u}(r, \theta, \pm \Gamma / 2, t) & =(0,0,0) \\
\boldsymbol{u}(1, \theta, z, t) & =(0,0, \text { Re } \sin (2 \pi S t t)) .
\end{aligned}
$$

The amplitude of the vertical oscillation of the cylinder wall, $\Delta z$, in terms of the non-dimensional parameters of the problem is given by $\Delta z=R e /(2 \pi S t)$, where $\Delta z$ has been non-dimensionalized using the radius $R$.

Since the flow is incompressible of constant density, the oscillating sidewall problem is identical to having a stationary sidewall with the top and bottom endwalls oscillating together in the axial direction. These idealized boundary conditions are discontinuous at the junctions where the stationary lids meet the oscillating sidewall. In a physical experiment, there are small but finite gaps at these junctions where the axial velocity adjusts rapidly to zero. For a proper use of spectral techniques, a regularization of this discontinuity is implemented of the form

$$
w(1, \theta, z, t)=\operatorname{Re} \sin (2 \pi S t t)\left[1-\exp \left(-\frac{\Gamma / 2-z}{\epsilon}\right)\right]\left[1-\exp \left(-\frac{\Gamma / 2+z}{\epsilon}\right)\right],
$$

where $\epsilon$ is a small parameter that mimics the small physical gaps $\left(\epsilon=6 \times 10^{-3}\right.$ has been used as a fixed parameter). The use of $\epsilon \neq 0$ regularizes the otherwise discontinuous boundary conditions; see Lopez \& Shen (1998) for further details on the use of this technique in spectral codes.

The governing equations and boundary conditions are invariant to the following spatial symmetries:

$$
\begin{aligned}
& K_{\theta}(u, v, w)(r, \theta, z, t)=(u,-v, w)(r,-\theta, z, t), \\
& R_{\alpha}(u, v, w)(r, \theta, z, t)=(u, v, w)(r, \theta+\alpha, z, t),
\end{aligned}
$$

for any real $\alpha . K_{\theta}$ represents reflections about any meridional plane, whilst $R_{\alpha}$ signifies rotations about the cylinder axis. $K_{\theta}$ and $R_{\alpha}$ generate the groups $Z_{2}$ and $S O(2)$, but the two operators do not commute, so the symmetry group generated by $K_{\theta}$ and $R_{\alpha}$ is $O(2)$ and it acts in the periodic azimuthal $\theta$-direction. The horizontal reflection on the mid-plane $z=0$ acts on the velocity field as

$$
K_{z}(u, v, w)(r, \theta, z, t)=(u, v,-w)(r, \theta,-z, t) .
$$

Due to the harmonic oscillation of the sidewall, the boundary condition $(2.5 b)$ is not $K_{z}$-invariant. However, the axial velocity on the sidewall satisfies $K_{z} w(1, \theta, z, t)=$ $w(1, \theta, z, t+\tau / 2)$, and the system is invariant to the spatio-temporal symmetry consisting of a reflection about the mid-plane $z=0$ together with a half-period evolution in time

$$
H(u, v, w)(r, \theta, z, t)=(u, v,-w)(r, \theta,-z, t+\tau / 2) .
$$

The transformation $H$ generates another $Z_{2}$ symmetry group that commutes with $O(2)$. Hence, the complete symmetry group of the problem is $Z_{2} \times O(2)$. The action of the spatial symmetry $K_{\theta}$ and the spatio-temporal symmetry $H$ on the vorticity is different to the action on the velocity (while the action of the rotations $R_{\alpha}$ is the same 
on the velocity and vorticity fields), and is given by

$$
\begin{aligned}
K_{\theta}(\xi, \eta, \zeta)(r, \theta, z, t) & =(-\xi, \eta,-\zeta)(r,-\theta, z, t), \\
H(\xi, \eta, \zeta)(r, \theta, z, t) & =(-\xi,-\eta, \zeta)(r, \theta,-z, t+\tau / 2) .
\end{aligned}
$$

Therefore, the individual symmetries (and the generated groups) are exactly the same as for the periodically driven annular cavity and analogous to the two-dimensional time-periodic wake of symmetrical bluff bodies and periodically driven rectangular cavity flows.

In the symmetry $Z_{2} \times O(2)$ corresponding to the problem considered, the spatial part of the spatio-temporal symmetry commutes with the purely spatial symmetries, which simplifies the analysis. However, it should be noted that in other similarly periodically forced hydrodynamic systems, the spatial parts of the spatio-temporal symmetry do not commute, and care needs to exercised; see Lopez \& Marques (2000), Marques \& Lopez (2000), Avila et al. (2007), Avila et al. (2008) and references therein.

\subsection{Numerical formulation}

The governing equations have been solved using a second-order time-splitting method. The spatial discretization is via a Galerkin-Fourier expansion in $\theta$ and a Chebyshev collocation in $x=2 r$ and $z$, of the form

$$
F(r, \theta, z)=\sum_{l=0}^{n_{r}} \sum_{n=0}^{n_{z}} \sum_{m=-n_{\theta}}^{n_{\theta}} a_{l, n, m} T_{l}(x) T_{n}(z) \mathrm{e}^{\mathrm{i} m \theta}
$$

for the three velocity components and pressure. The results presented here were computed with $n_{r}=48, n_{z}=96$ and $n_{\theta}=10$. This resolution resolves all the spatial scales in the solutions presented here. Time steps of $\delta t=10^{-5}$ have been required to ensure numerical stability and accuracy of the temporal scheme. For each Fourier mode, the corresponding Helmholtz and Poisson equations are solved efficiently using a complete diagonalization of the operators in both the radial and axial directions. Note that to decouple the Helmholtz equations for $u$ and $v$, we have used the combinations $u_{+}=u+\mathrm{i} v$ and $u_{-}=u-\mathrm{i} v$. The coordinate singularity at the axis $(r=0)$ is treated by using the parity of the azimuthal Fourier components of the variables to satisfy the regularity conditions at the origin needed to solve the Helmholtz equations (see Mercader, Net \& Falqués 1991, for details). The spectral collocation solver used here is based on a previous scheme (Mercader, Batiste \& Alonso 2010) that has recently been tested and used in a wide variety of flows in enclosed cylinders (Marques et al. 2007; Lopez et al. 2007, 2009; Lopez \& Marques 2009).

\section{Basic states}

The basic flow, having all the symmetries of the problem, is always axisymmetric and time-periodic, synchronous with the forcing. The axial oscillations of the cylinder sidewall produce periodic Stokes-type boundary layers on the oscillating wall. These layers separate from the sidewall and move towards the cylinder axis after colliding with the endwalls to form rollers. These rollers are formed every half-period alternately on each endwall. The term roller refers to large-scale rotating flow structures with primarily azimuthal vorticity, $\eta$. Instantaneous contours of the streamfunction $(\psi$, such that $u=-1 / r \partial \psi / \partial z$ and $w=1 / r \partial \psi / \partial r$ ) are shown in the first row of figure 2 for four increasing values of the forcing frequency $S t$, and for amplitudes $R e$ very 
(a)
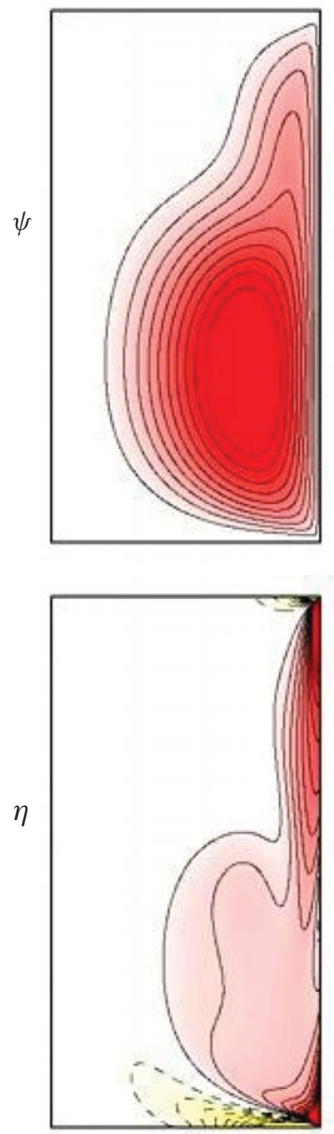

(b)
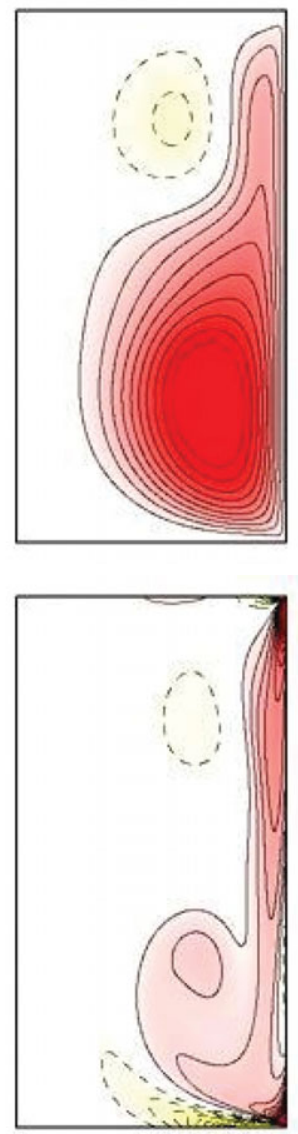

(c)
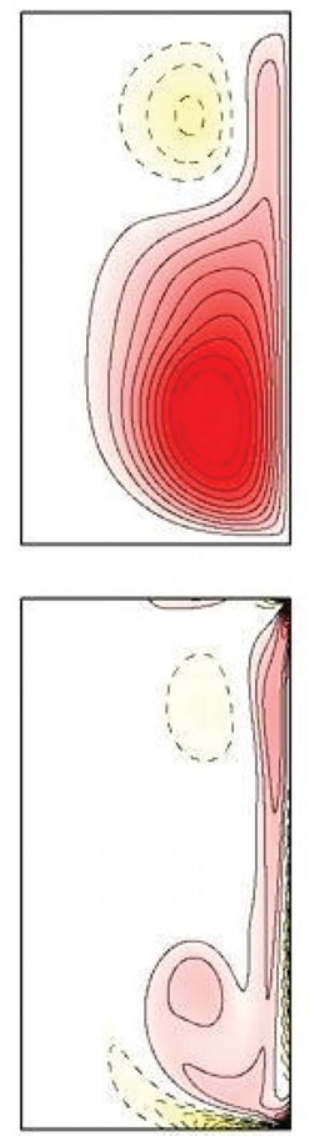

(d)
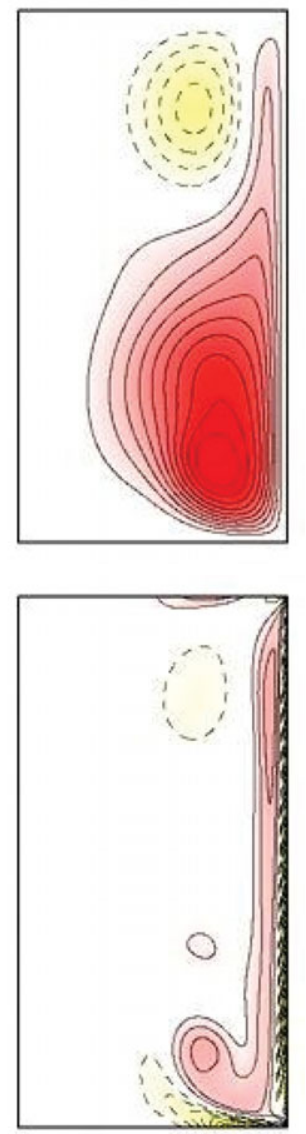

FIgURE 2. (Colour online) Contours of streamfunction, $\psi$, and azimuthal vorticity, $\eta$, of the basic state at four parameter values, for amplitudes $R e$ very close to the corresponding critical values: $(a)(S t, R e)=(10,340),(b)(S t, R e)=(32,525),(c)(S t, R e)=(50,615)$ and $(d)$ $(S t, R e)=(100,700)$. Solid (dashed) contours are positive (negative); light/dark (yellow/red online) colours correspond to negative/positive values. The base state is periodic, and we have selected for each $S t$ the phase of period where the oblique jet at the bottom corner is most intense; the supplementary movie 1 (available at journals.cambridge.org/FLM) shows temporal evolution over one period.

close to, and above, the critical value at which the basic flow becomes unstable; supplementary movie 1 shows these contours over a forcing period. These unstable basic states were computed in the axisymmetric subspace. In all cases, the figures represent meridional planes $(r, z) \in[0, r] \times[-\Gamma / 2, \Gamma / 2]$ and the sidewall is at rest at $t=0$.

The magnitude and size of the rollers changes substantially with St. For small forcing frequencies, there is sufficient time for these rollers to dissipate during part of the forcing period, and so in figure 2(a) a single roller fills the whole domain most of the time, whereas for large frequencies the rollers persist at both ends throughout the whole forcing cycle. The Stokes number determines the size of the rollers and their dissipation, and the Reynolds number measures the strength of their collision with the lids and the recirculation of the fluid. The characteristics of the rollers are similar to 
the ones described in previous works for the planar case (Blackburn \& Lopez 2003b) and for an annular cavity (Blackburn \& Lopez 2011), but in the present analysis the curvature effects are very important, and the flow geometry is substantially altered near the cylinder axis.

Instantaneous contours of the azimuthal vorticity are shown in the second row of figure 2. These contours describe the boundary layers that form at the sidewall and endwalls and their dynamics very well. The sidewall boundary layer is a Stokestype boundary layer whose thickness is proportional to $S t^{-1 / 2}$ (Schlichting \& Kestin 1979; Marques \& Lopez 1997), so the boundary layer becomes thinner for larger values of the forcing frequency $S t$ (it also becomes thinner as the amplitude of the forcing $R e$ is increased). The sidewall boundary layer, dragged by the cylinder sidewall motion, separates upon colliding with the endwalls, and from the corners where the sidewall and endwalls meet, the boundary layer enters the bulk of the fluid, forming axisymmetric oblique jets that result in the formation of the rollers. This process is analogous to the formation of a vortex roller near the junction of an impulsively started plate and a stationary plate, where there is a jump in the velocity (Allen \& Lopez 2007). The jets are clearly seen in the azimuthal vorticity contours: the jet centreline coincides with the azimuthal vorticity zero contour, and on each side it is surrounded by regions with intense azimuthal vorticity of opposite signs. Oscillating boundary layers also form on the endwalls with azimuthal vorticity of opposite sign to that of the rollers, as a result of the jet dynamics just described, and because the endwalls are at rest.

\section{Stability of the basic flow}

By increasing the amplitude of the forcing $R e$ beyond a critical value $R_{c}(S t)$, the basic state undergoes a symmetry-breaking bifurcation yielding a new threedimensional state. Depending on $S t$, the basic state may undergo either synchronous or Neimark-Sacker bifurcations. The stability of the basic flow has been comprehensively explored for $S t \in[1,150]$, revealing two synchronous modes $(A$ and $B)$ that bifurcate from the axisymmetric state by breaking the symmetries differently in each case. There is also a novel quasi-periodic $(Q P)$ mode that manifests as modulated rotating waves $M R W$. We use subscripts for each of these states to indicate their azimuthal wavenumber $m$.

The linear stability of the basic state to general three-dimensional perturbations has been determined using global linear stability analysis via time evolution of the Navier-Stokes equations (Lopez et al. 2009; Do, Lopez \& Marques 2010). First, a periodic axisymmetric basic state was computed at some point in parameter space. Its stability was determined by introducing small random perturbations into all azimuthal Fourier modes. For sufficiently small perturbations, the nonlinear couplings between Fourier modes are negligible (below round-off numerical noise) and the growth rates (real parts of the eigenvalues) and structure of the eigenfunctions corresponding to the fastest growing perturbation at each Fourier mode emerge from time evolution. This is tantamount to a matrix-free generalized power method in which the actions of the Jacobian matrices for the perturbations are given by time integration of the NavierStokes equations with the aforementioned initial conditions. This direct numerical technique is very efficient as the exponential growth or decay of the perturbations is established in a relatively short evolution time, and there is no need to evolve the disturbances until they saturate nonlinearly. The technique has been used on a variety of fluid dynamics problems where the state whose stability is being analysed is 


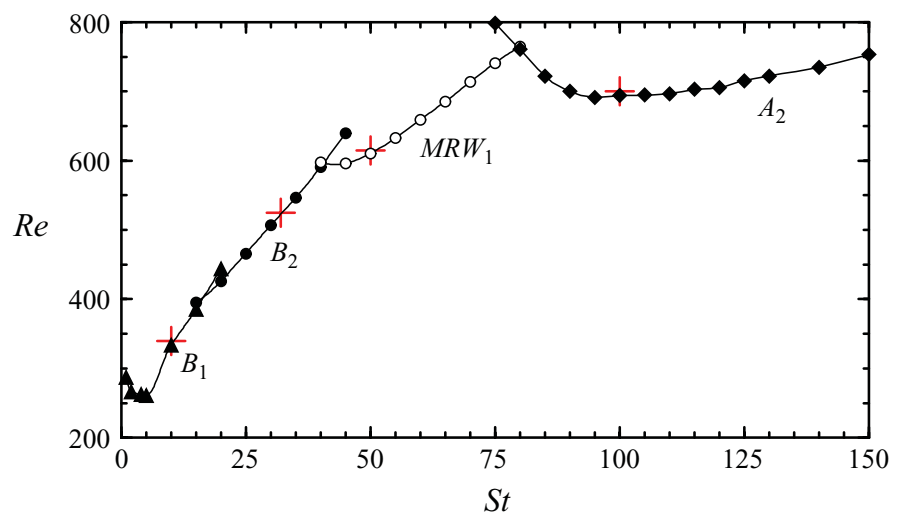

Figure 3. (Colour online) Critical Reynolds number, $R e_{c}$, as a function of the Stokes number, $S t$, for the transition from the basic state to the different three-dimensional states, $B_{1}, B_{2}$, $M R W_{1}$ and $A_{2}$. The crosses (red in the online version) correspond to the four basic states depicted in figure 2 .

non-trivial (see Lopez \& Marques 2009; Lopez et al. 2009; Rubio, Lopez \& Marques 2010, for examples and some further details).

The bifurcation curves for the different modes in $(S t, R e)$-space are shown in figure 3. At low $S t$, mode $B$ is the first to become critical with increasing $R e$, while at high $S t$ mode $A$ is the first. At intermediate values $S t \in[40.7,79.7]$, the quasi-periodic mode bifurcates first, in the form of modulated rotating waves $M R W$. The synchronous mode $A$ always has an azimuthal wavenumber $m=2\left(A_{2}\right)$, the quasi-periodic mode has $m=1\left(M R W_{1}\right)$ and the synchronous mode $B$ may have either $m=1$ or $m=2$ depending on $S t$. The bifurcations to the four different states $\left(B_{1}, B_{2}, M R W_{1}\right.$ and $A_{2}$ ) when varying the forcing frequency $S t$ are separated by three codimension-two bifurcation points at which two of the states bifurcate simultaneously. The four base states shown in figure 2 correspond to the four distinct bifurcated states in figure 3 . The synchronous modes for small $S t$ have azimuthal wavenumber $m=1\left(B_{1}\right)$ and a single roller fills the domain most of the time, whereas they have azimuthal wavenumber $m=2\left(B_{2}\right.$ and $\left.A_{2}\right)$ for larger $S t>15$ and two rollers persist throughout the whole forcing cycle. However, the quasi-periodic $M R W_{1}$ has azimuthal wavenumber $m=1$ although it is dominant in an $S t$ region where the two rollers persist.

A very similar scenario occurred in the periodically driven rectangular cavity problem, as illustrated in figure 4, showing the critical Re as a function of $S t$ in the cavity flow (adapted from Blackburn \& Lopez 2003b; Leung et al. 2005). Figures 3 and 4 are strikingly similar, and they only differ in their scaling. The critical $R e$ and $S t$ for the rectangular cavity are about a factor of 2 larger than for the cylinder case, so that the marginal curve in the cavity flow occurs at higher $R e$, and the different modes are shifted to higher St. The qualitative shape of the marginal curves is very similar in both the cases, and the shift in $(S t, R e)$ reflects the different geometries of the two problems. An important difference between the two problems is that in the driven rectangular cavity, the wavenumber of the bifurcated solution varies continuously, while in the cylinder problem it is discrete (and, in fact, of very small wavenumber, either $m=1$ or $m=2$ ). However, the qualitative trend is the same in both problems. In the driven rectangular cavity, the wavenumber of mode $B$ increases with $S t$, while for $Q P$ and $A$ their wavenumbers are almost independent of St (Leung et al. 2005). 


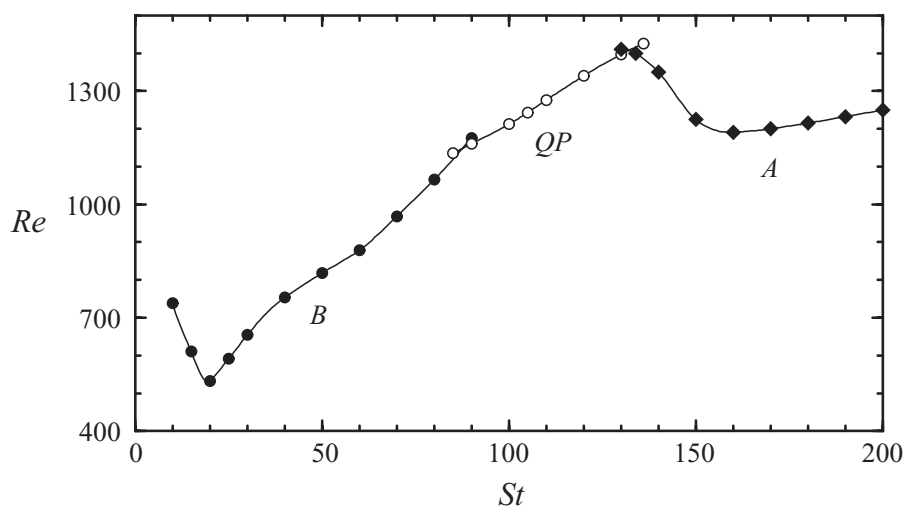

Figure 4. Critical Reynolds number, $R e_{c}$, as a function of the Stokes number, St, for the transition from the basic state to the different three-dimensional states, $B, Q P$ and $A$, for the periodically forced rectangular cavity flow (adapted from Blackburn \& Lopez 2003b; Leung et al. 2005).

In the cylinder problem, the azimuthal wavenumber of mode $B$ also increases with $S t$ (varying from $m=1$ to $m=2$ ), while for $M R W$ and $A$ their azimuthal wavenumbers do not vary with $S t$ ( $m=1$ for $M R W$ and $m=2$ for $A$ ).

A detailed comparison with the annular cavity problem (Blackburn \& Lopez 2011) is not possible for a number of reasons. That study focused on the modulated rotating waves at a single value of $S t=100$, and at that value of $S t$ the $M R W$ were found to be unstable. The nonlinear flows were complicated, showing mixed characteristics between the synchronous and quasi-periodic modes. Nevertheless, the different modes obtained in the present cylinder problem were also present in the annular cavity problem. The radius ratio used in the annular study was close to 1 , so that both inner and outer radii were much larger than the annular gap. That choice of the radius ratio was made to compare with the rectangular cavity flow problem, which corresponds to the radius-ratio-going-to-1 limit. As a result, the azimuthal wavenumbers of the bifurcating states were very large (between $m=30$ and $m=35$ for the dominant modes in the parameter regime considered). In contrast, in the cylinder problem, which corresponds to radius ratio equal to 0 , the azimuthal wavenumbers are very small $(m=1$ and $m=2)$. Furthermore, even though the two problems have the same symmetry group, the flow domain in the cylinder is singly connected whereas in the annulus it is doubly connected.

\section{Three-dimensional structure and symmetries of the unstable modes}

After perturbing the axisymmetric basic flow with $R e>R e_{c}(S t)$, a new threedimensional periodic or quasi-periodic state is reached, after waiting enough time for saturation. This bifurcated state depends strongly on the mode that drives the instability, and on the precise values of $(S t, R e)$. When describing these bifurcated flows, we will use the term braid, widely used in similar flows, to denote smaller scale meridional structures with vorticity components $\xi$ and $\zeta$. Braids are typically generated through the amplification of spanwise-orthogonal perturbations of the rollers in rectangular cavities, and in cylindrical and annular geometries it is the amplification of meridional perturbations of the rollers that gives rise to the braids. 

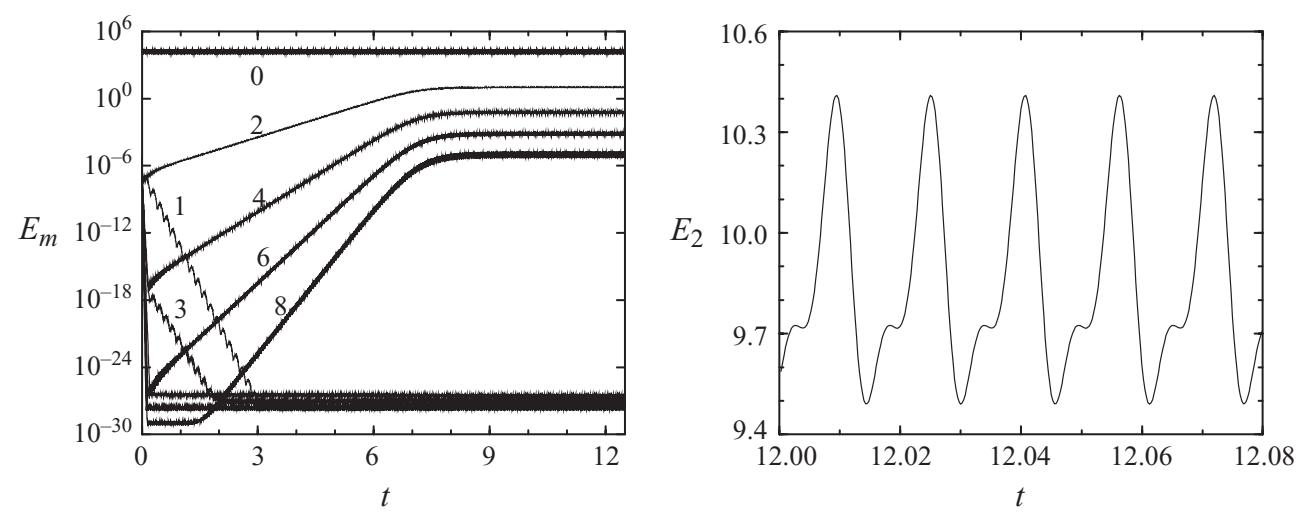

FIgURE 5. (a) Time series of the energies of the leading Fourier modes, with azimuthal wavenumbers $m$ as indicated, for the $B_{2}$ state at $R e=525$ and $S t=32$; and $(b)$ a close-up of $E_{2}(t)$ after saturation.

In the following subsections, the symmetries and features of the different bifurcated solutions are described and illustrated with results computed at given values of $S t$. Modes $B_{1}, B_{2}$ and $A_{2}$ are computed at $S t=10,32,100$, respectively, whilst mode $M R W_{1}$ has been computed at $S t=50$, and the corresponding base states have already been illustrated in figure 2. All the solutions have been computed at $R e$ slightly above $R e_{c}$.

\subsection{Synchronous modes}

Three-dimensional synchronous states result when a pair of purely real eigenvalues (multiplicity 2 due to the $O(2)$ symmetry) cross the unit circle at +1 in the complex plane. When a synchronous axisymmetric flow is perturbed, the energies of the Fourier modes may grow or decay depending on the case, but regardless their evolution is clearly modulated by the sidewall frequency. When the basic flow is unstable to synchronous modes, the Fourier spectra begin to grow and after some time reach an asymptotic state where the modes are saturated but oscillate with the driving frequency of the sidewall about a mean value. Such an evolution can be seen in figure 5, where the energies, $E_{m}$, of the leading Fourier modes are shown as a function of time for the $B_{2}$ state at $R e=525$ and $S t=32$; figure $5(b)$ shows the oscillations in the energy, synchronous with the forcing (period $\tau=1 / S t=0.03125$ ), but with the period halved because the energy is a sum of squares of the velocities.

The three-dimensional structures of modes $A$ and $B$ are visualized in figure 6 with the aid of perspective views of instantaneous isosurfaces of the radial vorticity (dark/light, or yellow/red in supplementary movie 2, are positive/negative values), which show the braid structures, and azimuthal vorticity (translucent), showing the rollers. Note that the only component of vorticity of the axisymmetric base state that is non-zero is the azimuthal component, and that the braids comprise radial and axial components of vorticity and are a direct result of breaking axisymmetry. In general terms, braids are located near the lids and away from the sidewall, and they are born on the oblique jets alternately emerging from the top and bottom corners. Nevertheless, there are some subtle variations. For $A_{2}$, braids suffer slight changes in shape and their behaviour is quite regular as is that of the rollers. Notice that the shape of each roller is essentially the same as those of the base state. For $B_{1}$ and $B_{2}$, braids change abruptly during a forcing cycle, as do the rollers in this regime, 
(a)

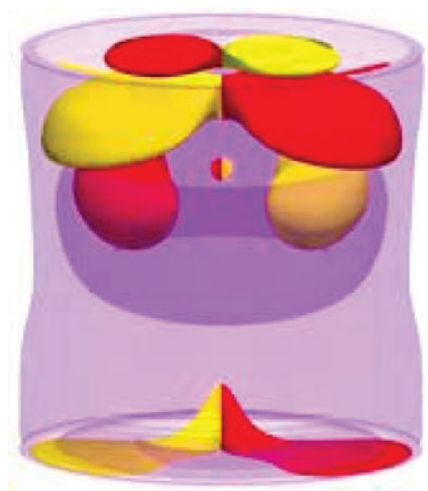

(b)

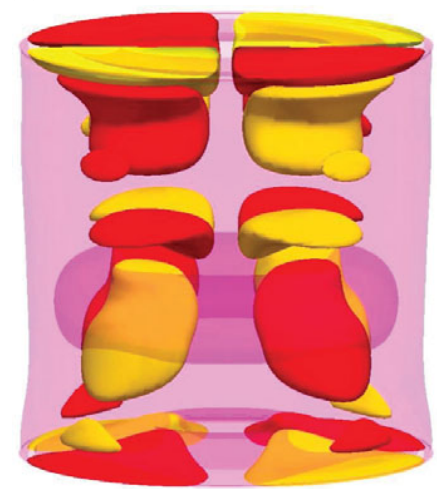

(c)

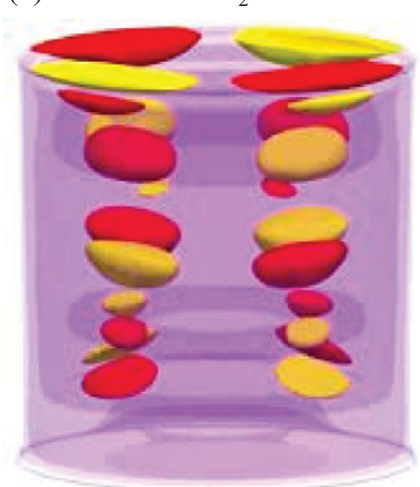

Figure 6. (Colour online) Isosurfaces of radial vorticity $\xi$ (solid) and azimuthal vorticity $\eta$ (translucent) for the synchronous states $(a) B_{1}$ at $(R e, S t)=(340,10)$ with $\xi= \pm 50$ and $\eta= \pm 500,(b) B_{2}$ at $(R e, S t)=(525,32)$ with $\xi= \pm 40$ and $\eta= \pm 1000$ and $(c) A_{2}$ at $(R e, S t)=(700,100)$ with $\xi= \pm 150$ and $\eta= \pm 800$. Supplementary movie 2 shows temporal evolution over one forcing period.

and their dynamics (creation, merging and destruction) is much more complex. In addition, the azimuthal vorticity of the $B$ modes is very different from that of the corresponding basic state.

As the bifurcated solutions are no longer axisymmetric, the $O(2)$ symmetry has been broken. Of the continuous family of rotations $R_{\alpha}$, there only remains the discrete symmetry $R_{2 \pi / m}$, a rotation of angle $2 \pi / m$ around the axis and its powers. The azimuthal wavenumber of the bifurcated solution is $m$. The continuous family of reflection symmetries about meridional planes, $K_{\theta}$, is also reduced to a collection of $m$ reflection planes at angles $\pi / m$ apart, as was shown in Marques et al. (2004). These spatial symmetries are clearly evident in figures 7 and 8 . The planes of reflection symmetry correspond to the diameters with zero axial vorticity. Note that when the symmetry is preserved, the axial vorticity changes sign according to $(2.10 a)$. The rotation and meridional reflection symmetries just described generate the so-called $D_{m}$ symmetry group (or dihedral group) with $2 m$ elements, consisting of $m$ rotations and $m$ meridional reflections. When $m=1$, this group contains only the identity and one meridional reflection, and is isomorphic to $Z_{2}$.

Now, let us examine what happens with the spatio-temporal symmetry $H$. Figure 7 shows contours of the axial vorticity of the critical eigenvectors for the $B_{1}, B_{2}$ and $A_{2}$ bifurcations in a horizontal section $z=\Gamma / 4$ for a given time, and in the reflectionsymmetric section $z=-\Gamma / 4$ after advancing half the forcing period. The figure shows that the bifurcations to $B_{1}$ and $B_{2}$ are $H$-symmetric, i.e. the values of the axial vorticity $\zeta$ of the eigenfunctions, at a given time $t_{0}$ and at $z=\Gamma / 2$ (figure $7 a$ ), are the same as the values of $\zeta$ advancing time by half the forcing period, $t_{0}+\tau / 2$, on the reflection-symmetric plane $z=-\Gamma / 2$ (figure $7 b$ ). The eigenfunction of the $A_{2}$ bifurcation is not $H$-symmetric, but changes sign, so the $H$-symmetry is broken in this bifurcation. However, $H$ combined with the rotation $R_{\pi / m}$, with $m=2$ (half the angle of the rotational symmetry of the state), results in a space-time symmetry of the $A_{2}$ eigenfunction. This is precisely the expected behaviour from bifurcation theory (Marques et al. 2004): there are only two options for three-dimensional synchronous eigenfunctions under the action of the space-time symmetry $H$, either multiplication 

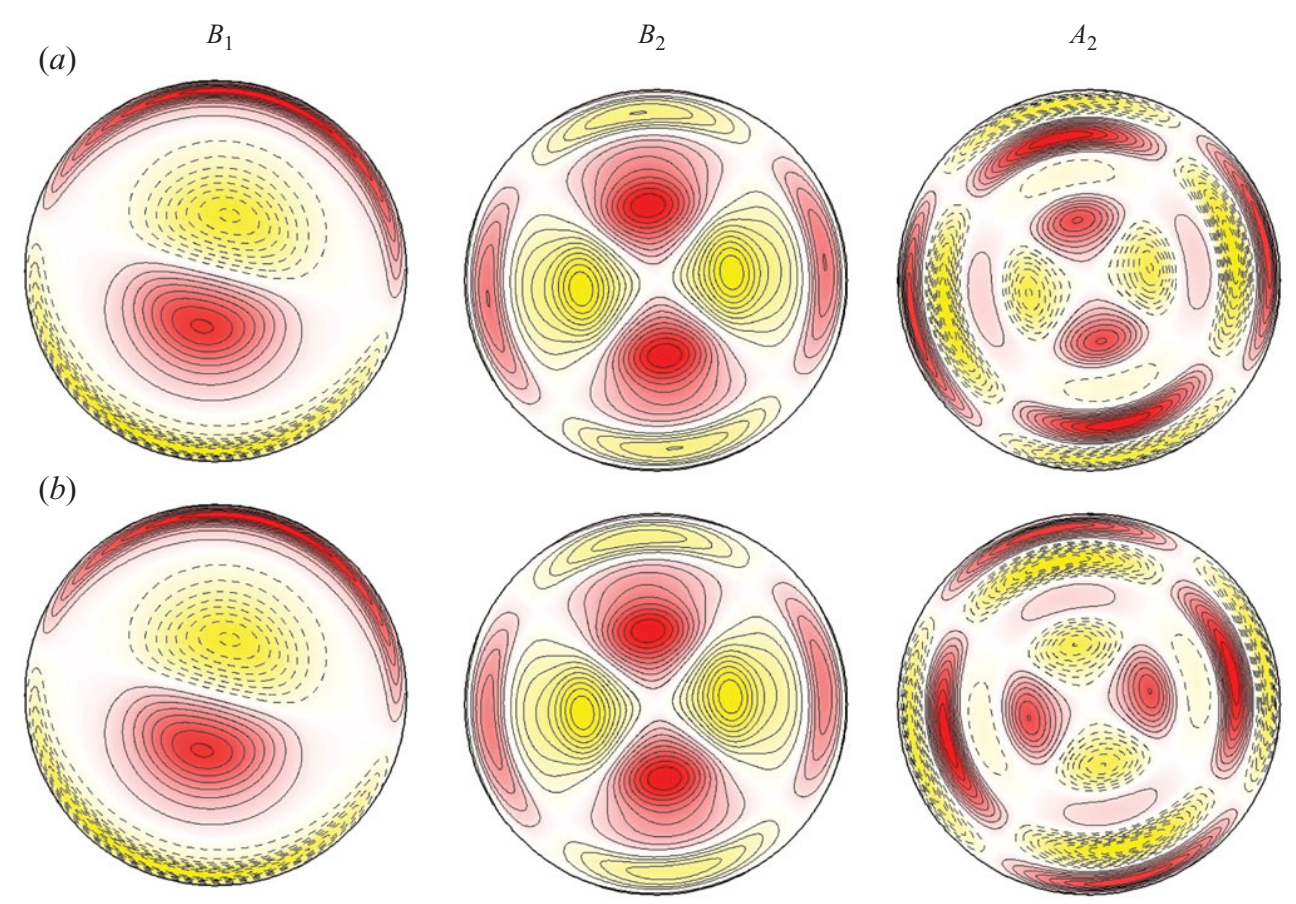

FIGURE 7. (Colour online) Axial vorticity contours in a $z$-constant plane of the eigenfunctions at the bifurcation to states $B_{1}, B_{2}$ and $A_{2}$ shown in figure $6 ;(a)$ is computed at a convenient time $t_{0}$ and $z=\Gamma / 4$, while $(b)$ is computed at time $t_{0}+\tau / 2$ and $z=-\Gamma / 4$, i.e. applying the space-time transformation $H$ to $(a)$. Solid (dashed) contours are positive (negative); light/dark (yellow/red online) colours correspond to negative/positive values.

by 1 or -1 . The behaviour of all the velocity and vorticity components is given by

$\begin{array}{ll}H \text { preserved: } & \left\{\begin{array}{l}(u, v, w)(r, \theta, z, t)=(u, v,-w)(r, \theta,-z, t+\tau / 2), \\ (\xi, \eta, \zeta)(r, \theta, z, t)=(-\xi,-\eta, \zeta)(r, \theta,-z, t+\tau / 2),\end{array}\right. \\ H \text { broken: }\left\{\begin{array}{l}(u, v, w)_{e}(r, \theta, z, t)=(-u,-v, w)_{e}(r, \theta,-z, t+\tau / 2), \\ (\xi, \eta, \zeta)_{e}(r, \theta, z, t)=(\xi, \eta,-\zeta)_{e}(r, \theta,-z, t+\tau / 2),\end{array}\right. \\ R_{\pi / m} H \text { preserved: }\left\{\begin{array}{l}(u, v, w)(r, \theta, z, t)=(u, v,-w)(r, \theta+\pi / m,-z, t+\tau / 2), \\ (\xi, \eta, \zeta)(r, \theta, z, t)=(-\xi,-\eta, \zeta)(r, \theta+\pi / m,-z, t+\tau / 2) .\end{array}\right.\end{array}$

The space-time symmetries of the eigenfunctions are inherited by the nonlinear saturated states (as long as no additional bifurcations take place in the saturation process). However, the multiplication by -1 , whose action is described by (5.2), is a property only of the eigenfunction and not of the resulting saturated nonlinear state. The reason is that the eigenfunctions are pure Fourier modes in the azimuthal direction, and when they develop into fully nonlinear three-dimensional bifurcated solutions, Fourier harmonics appear, and the even harmonics (including the zero mode) are multiplied by $(-1)^{2}=+1$ under the action of the $H$-symmetry, so the full nonlinear solution does not have the multiplication by -1 property, (5.2), that the 

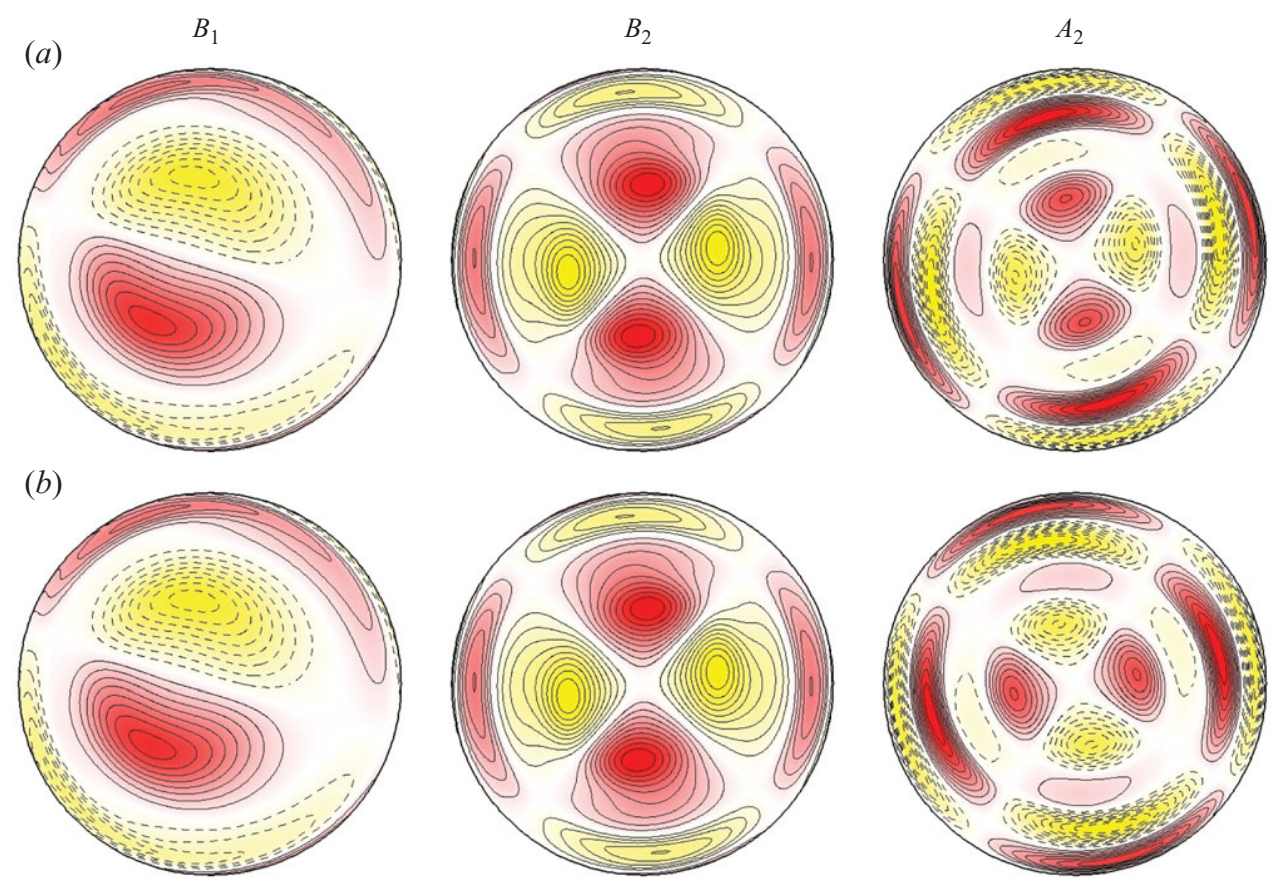

Figure 8. (Colour online) Axial vorticity contours in a $z$-constant plane of nonlinear saturated states corresponding to $B_{1}, B_{2}$ and $A_{2}$ shown in figure $6 ;(a)$ is computed at a convenient time $t_{0}$ and $z=\Gamma / 4$, while $(b)$ is computed at time $t_{0}+\tau / 2$ and $z=-\Gamma / 4$, i.e. applying the space-time transformation $H$ to $(a)$. Solid (dashed) contours are positive (negative); light/dark (yellow/red online) colours correspond to negative/positive values.

eigenfunction has. Figure 8 shows the same information as in figure 7 , but for the full nonlinear bifurcated solutions, illustrating the symmetry properties of the saturated states. It is only the eigenfunctions and not the nonlinear states that are invariant to the action (5.2), hence the use of the subscript $e$, signifying eigenfunction. The preserved symmetries $H(5.1)$ and $R_{\pi / m} H$ (5.3) clearly persist in the nonlinear states.

Synchronous modes $A$ and $B$ with azimuthal wavenumber $m$ have the same spatial symmetry group, the dihedral group $D_{m}$ and they also have one spatio-temporal symmetry: $H$ for the $B$ modes, and $R_{\pi / m} H$ for the $A$ modes. However, the complete symmetry groups of the bifurcated states are different: as $H$ commutes with $O(2)$, it also commutes with the subgroup $D_{m}$, and the complete symmetry group for the $B$ modes is the direct product $D_{m} \times Z_{2}$. However, $R_{\pi / m} H$ does not commute with $D_{m}$, because rotations and meridional reflections do not commute $\left(K_{\theta} R_{\alpha}=R_{-\alpha} K_{\theta}\right)$. So, for the $A$ modes, the complete symmetry group is the semi-direct product $D_{m} \rtimes Z_{2}$. This is an example of a complete symmetry group where the spatial and spatio-temporal symmetries do not commute, a possibility discussed just before $\$ 2.1$, in Marques $e t$ al. (2004), and also from a more theoretical point of view in Lamb (1996) and Lamb \& Melbourne (1999).

The three bifurcations to $B_{1}, B_{2}$ and $A_{2}$ are supercritical, as shown in figure 9 , where the time-averaged energy of the dominant mode $\left\langle E_{m}\right\rangle$ is plotted as a function of the Reynolds number. The plot in figure $9(a)$ is not linear, but this is not surprising as the path of increasing $R e$ in the $(R e, S t)$-parameter space is not normal to the bifurcation 

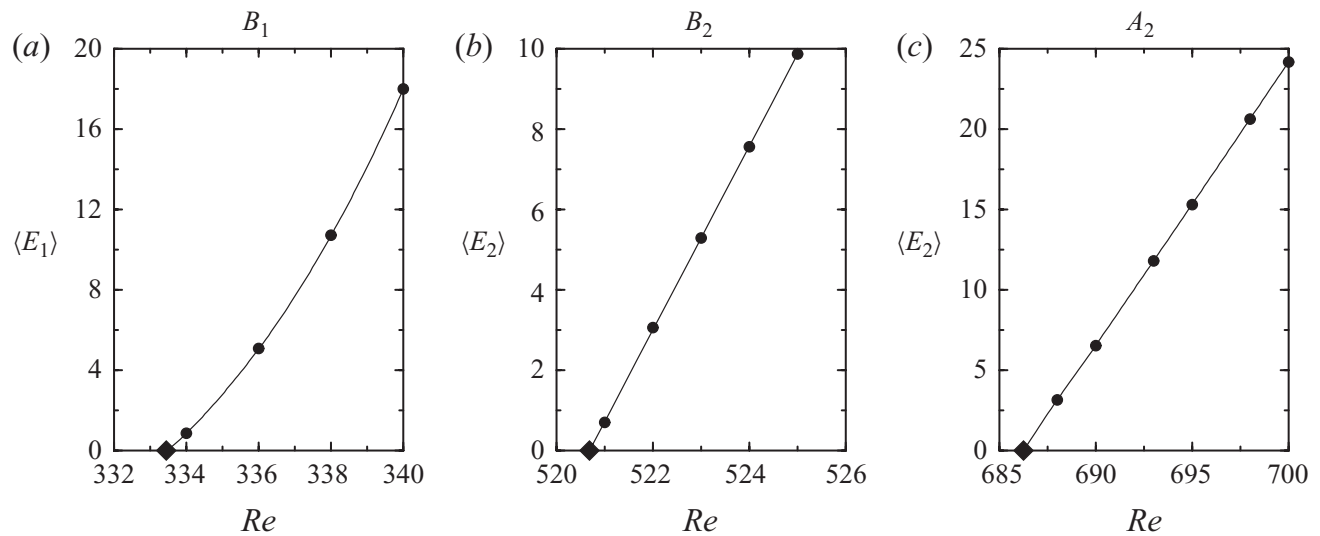

FIGURE 9. Time average of the energy of the dominant mode for the synchronous solutions as a function of $R e,(a) B_{1}$ solutions at $S t=10,(b) B_{2}$ solutions at $S t=$, and $(c) A_{2}$ solutions at $S t=100$. The diamond corresponds to the critical $R e$ obtained by linear stability analysis and the solid disks correspond to computed nonlinear solutions.

curve (see figure 2). There is no hysteresis and the behaviour of $\left\langle E_{m}\right\rangle$ is linear as $R e$ approaches the critical value $(\bullet)$. The normal form for the amplitude of the bifurcated synchronous solutions in the supercritical case is given by $\dot{A}=A\left(\mu-c|A|^{2}\right)$. When saturation is reached, $\dot{A}=0$ and we have $R e-R e_{c}=\mu=c|A|^{2}=d\left\langle E_{m}\right\rangle$, the observed linear behaviour close to the bifurcation point.

\subsection{Quasi-periodic mode}

The onset of the quasi-periodic states occurs when two complex-conjugate pairs of eigenvalues cross the unit circle, thus introducing a second frequency $\omega_{Q}$ related to the phase of the complex-conjugate pairs. Generically, this second frequency will be incommensurate with the forcing frequency, so the $H$-symmetry is broken in this bifurcation. The second frequency $\omega_{Q}$ can manifest itself in two ways, depending on whether the bifurcation breaks $K_{\theta}$ or $R_{\alpha}$ of the $O(2)$ symmetry of the basic state. In the linear stability analysis, we referred only to mode $M R W_{1}$, but this term encompasses modulated $\theta$-travelling wave $(M R W)$ and modulated standing wave $(M S W)$ states. Due to the $O(2)$ symmetry of the governing equations, there are two pairs of complex-conjugate eigenvalues that bifurcate simultaneously, and they correspond to modulated $\theta$-travelling waves that can travel in the positive or negative $\theta$-direction; after a period of forcing, the flow pattern repeats itself, but rotates a certain angle, $\pm \theta_{0}$, related to the second frequency by $\theta_{0}=2 \pi \omega_{Q} / S t$, where $S t=\omega_{f}$ is the forcing frequency. $K_{\theta}$ transforms each one of the $M R W$ into the other, therefore the $K_{\theta}$-symmetry is broken; the $S O(2)$ rotational symmetry is also broken, because the solution has azimuthal wavenumber $m=1$; however, as they are modulated travelling waves, there is a preserved space-time symmetry, consisting of advancing one forcing period in time combined with the rotation $R_{ \pm \theta_{0}}$.

Besides the two $M R W$ solutions, there is also a third nonlinear solution corresponding to a symmetric combination of the two $M R W$ states; these states, called modulated standing waves $M S W$, are $K_{\theta}$-symmetric, but the $S O(2)$ rotational symmetry is completely broken. Only one of the two families of solutions, $M R W$ and $M S W$, is stable (Marques et al. 2004). In the present problem, as in the case for the driven annular cavity (Blackburn \& Lopez 2003b) and for the rectangular-driven cavity (Leung et al. 2005), the stable solutions are $M R W$; their sense of travel depends 
$(a)$

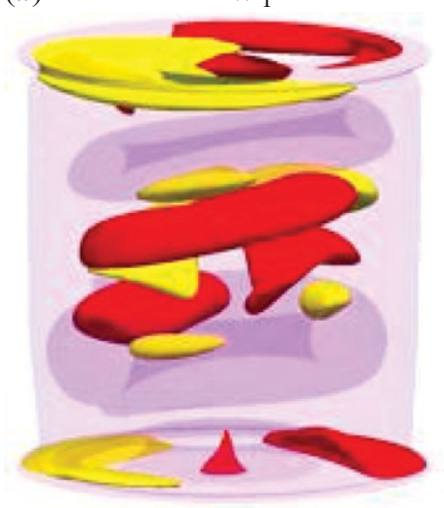

(b)

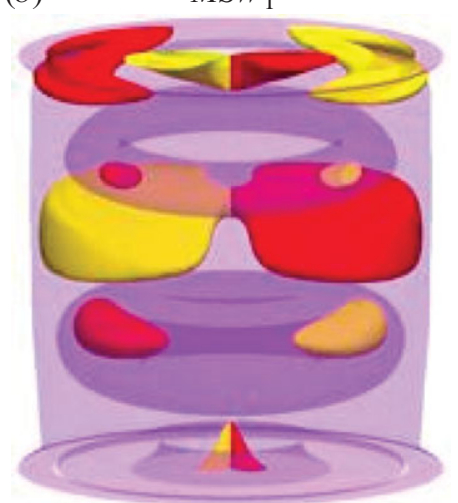

FiguRE 10. (Colour online) Isosurfaces of radial vorticity $\xi$ (solid) and azimuthal vorticity $\eta$ (translucent) for (a) $M R W_{1}$ with $\xi= \pm 180$, and $(b) M S W_{1}$ with $\xi= \pm 120$, both with $\eta= \pm 1000$ and computed at $(R e, S t)=(615,50)$; only $M R W_{1}$ is stable. Light $/$ dark (yellow/red online) solid colours correspond to negative/positive values of $\xi$. Supplementary movie 3 shows temporal evolution over several forcing periods.

on the initial condition for the sign of the azimuthal velocity perturbation. In order to obtain $M S W$, it is necessary to enforce the $K_{\theta}$ symmetry, restricting the computations to the appropriate Fourier subspace.

When an axisymmetric flow unstable to $M R W_{1}$ mode is perturbed, the energies of all Fourier modes begin to grow with the driving frequency and this is additionally modulated by the quasi-periodic frequency, which is approximately one order of magnitude smaller than the forcing frequency, and it is the same for $M R W$ and $M S W$ states. However, when $M S W$ reaches a saturated state, the energies of the Fourier modes retain both characteristic times, whilst the quasi-periodic frequency for $M R W$, being related to the azimuthal precession of the pattern, does not manifest in the energy of the Fourier modes.

The three-dimensional structures of these quasi-periodic flows are visualized in figure 10 by means of perspective views of instantaneous isosurfaces of the axial vorticity (dark/light are positive/negative values) and azimuthal vorticity (translucent). Braids are concentrated on the cylinder endwalls away from the sidewall and suffer large variations in all cases. As for the synchronous modes, the braids seem to be born along the oblique jets emerging from the corners and propagate into the interior, interacting in a complex way with the braids coming from the other endwall. For $M S W$, the braids possess very regular shapes and look quite similar to those of the synchronous states, and the rollers are virtually not distorted. For $M R W$, however, the braids have a helical structure and the rollers do not resemble the corresponding base flow rollers at all. In fact, the rollers in $M R W$ are tilted with respect to the horizontal rollers in the base state.

Figure 11 shows the same contours as in figure 10, all at the same phase at integer multiples of the forcing period apart. For $M R W$, the strobed structures do not change in a frame of reference that rotates in azimuth an angle $\theta_{0}$ each forcing period, justifying the name of modulated rotating wave. For the $M R W$ shown in figures 10 and 11 at $(R e, S t)=(615,50)$, this value is $\theta_{0} \approx 28.77^{\circ}$. This results in a precession frequency $\omega_{p}=3.996$. For $M S W$, the strobed structures vary substantially in one period, as can be seen in supplementary movie 4 associated with 

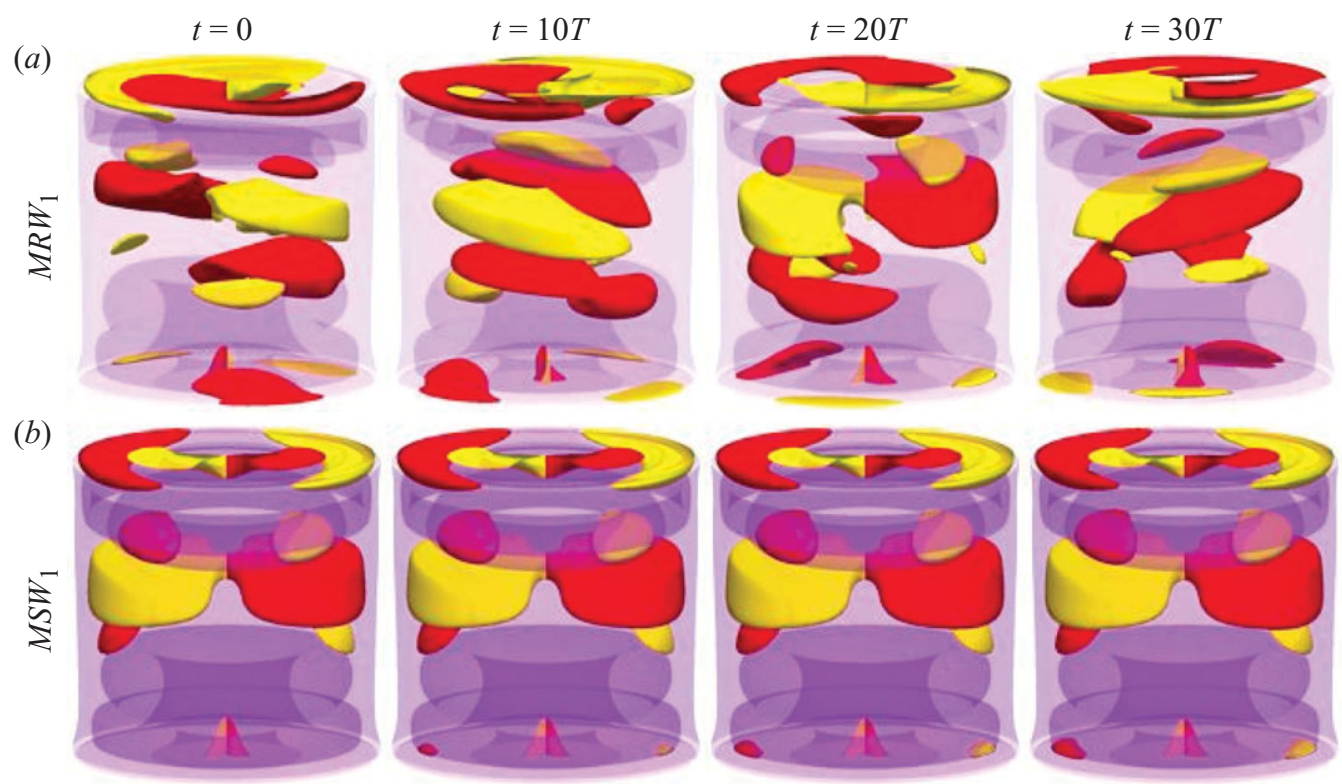

Figure 11. (Colour online) Same solutions and isosurface levels as in figure 10, but strobed every 10 forcing periods. Supplementary movie 4 shows temporal evolution strobed every forcing period.

figure 11(b). In general, the ratios between the quasi-periodic and sidewall periods are not commensurate, and the flow structure never repeats itself. However, the flow structure of $M S W$ remains almost unchanged after 10 forcing periods, as shown in figure $11(b)$; the only noticeable difference is the formation of braids very close to the bottom lid. This is because the ratio of quasi-periodic to forcing frequencies is very close to $1 / 10$ for the parameter values $(R e, S t)=(615,50)$ of $M S W$; this will be explored in more detail at the end of the present section.

The quasi-periodic bifurcation is subcritical, for both $M R W$ and $M S W$, in contrast to the synchronous bifurcations which are supercritical. Figure 12 shows the time average of the energy of the $m=1$ dominant mode for the quasi-periodic solutions, $\left\langle E_{1}\right\rangle$, as a function of $R e$. The $M R W$ solutions show a well-defined hysteretic region; for $M S W$, the hysteretic region is very costly to compute as there are extremely long transients. The energies do not behave linearly on the branch close to the critical Reynolds number, $R e_{c}=610.47$ for $S t=50$. We can fit the computed energy amplitudes to the shape predicted by normal form theory. According to Marques et al. (2004), the amplitudes of the bifurcated solutions vary as

$$
R e-R e_{c}=\mu=a|A|^{4}-b|A|^{2}=a\left\langle E_{1}\right\rangle^{2}-b\left\langle E_{1}\right\rangle,
$$

where a quartic term has been included due to the subcritical nature of the bifurcation. The fitting parameters $a$ and $b$ can be expressed in terms of the energy and Re at the saddle-node point

$$
\frac{R e-R e_{c}}{R e_{c}-R e_{S N}}=\frac{\left\langle E_{1}\right\rangle}{\left\langle E_{1}\right\rangle_{S N}}\left(\frac{\left\langle E_{1}\right\rangle}{\left\langle E_{1}\right\rangle_{S N}}-2\right) .
$$

The solid lines in figure 12 are best fits of this expression to the computed values. The agreement is very good and provides good estimates of the Reynolds numbers 


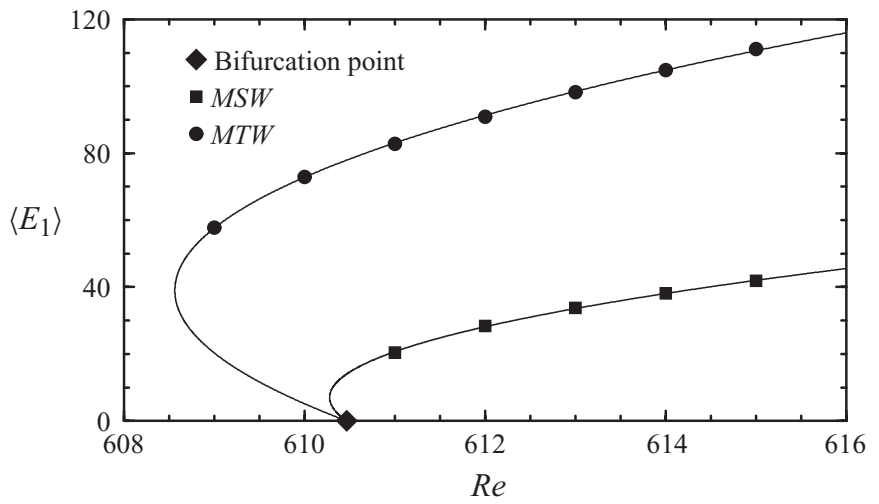

FIGURE 12. Time average of the energy of the $m=1$ dominant mode for the quasi-periodic solutions as a function of $R e$, for $S t=50$. The diamond corresponds to the critical $R e$ obtained by linear stability analysis. Symbols correspond to computed saturated solutions, and the curves are best fits to the parabolic profiles predicted by the normal form theory.
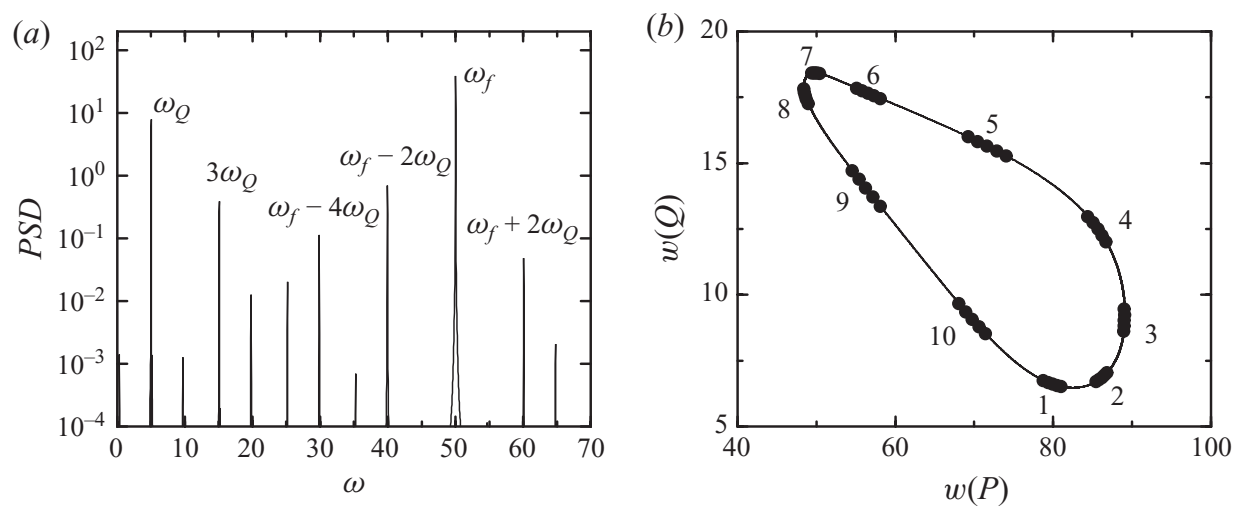

Figure 13. (a) Power spectral density of the FFT for $M S W$ at $(R e, S t)=(615,50)$, and (b) a Poincare section of the same solution strobing $M S W$ every $\tau$. Symbols $\bullet$ and numbers in $(b)$ correspond to successive iterates, showing that the frequency ratio is close to rational: $\omega_{Q} / \omega_{f} \approx 1 / 10$.

of the saddle-node bifurcations. The estimates are $R e_{S N} \approx 610.28$ for $M S W$ and $R e_{S N} \approx 608.57$ for $M R W$.

The frequencies of the quasi-periodic states can be computed via fast Fourier transform (FFT) of the time series of a convenient variable; here, we have chosen the value of the axial velocity at a point $P$ close to the sidewall at the cylinder midplane, $(r, \theta, z)_{P}=(0.9,0,0)$. Figure $13(a)$ shows the power spectral density for $M S W$ at $(R e, S t)=(615,50)$. It is a quasi-periodic spectrum with two well-defined frequencies, $\omega_{f}=50$ and $\omega_{Q}=5.035$, and their linear combinations. The ratio of the frequencies is close to resonance, $\omega_{Q} / \omega_{f}=0.1007 \approx 1 / 10$, and so a small frequency corresponding to $10 \omega_{Q}-\omega_{f} \approx 0.353$ is also present. The FFT supplies $\omega_{Q}$ up to a multiple of the forcing frequency; its precise value must be obtained by other methods. In order to analyse in detail how close to resonance the Neimark-Sacker bifurcation is and also to confirm the value of the second frequency, we have computed Poincare sections of the quasi-periodic states by strobing the $M S W$ solution every period $\tau$. The Poincare section is shown in figure $13(b)$, where we have projected the infinite-dimensional 
(a)

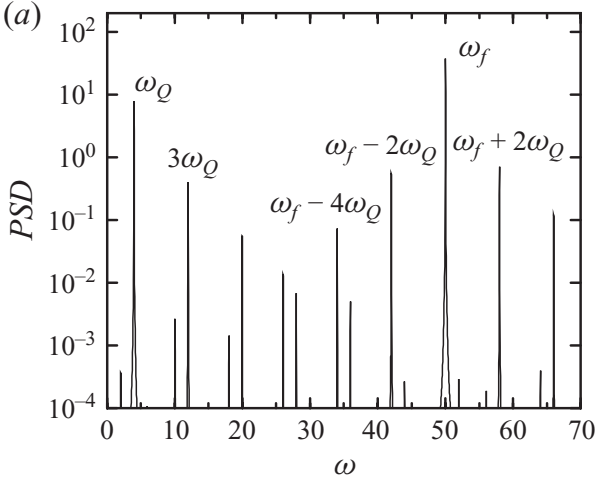

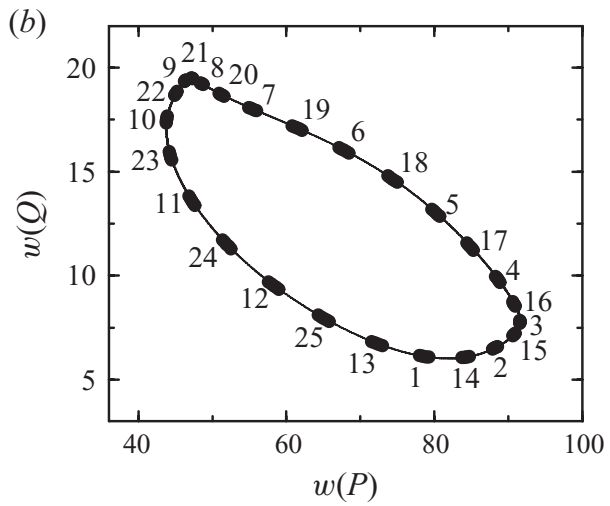

FIGURE 14. (a) Power spectral density of the FFT for $M R W$ at $(R e, S t)=(615,50)$, and (b) a Poincare section of the same solution strobing $M R W$ every $\tau$. Symbols $\bullet$ and numbers in $(b)$ correspond to successive iterates, showing that the frequency ratio is close to rational: $\omega_{Q} / \omega_{f} \approx 2 / 25$.

phase space into the plane corresponding to the values of the axial velocity $w$ at two different locations $P$ and $Q$ in the cylindrical domain. The point $P$ is the same as used for computing the FFT, at $(r, \theta, z)_{P}=(0.9,0,0)$, and $Q$ is close to the bottom endwall at $(r, \theta, z)_{P}=(0.9,0,-0.1)$. The closed curve is the section of the two-torus where the solution lives, and the symbols $\bullet$ correspond to successive iterates that are numbered in the figure. The 10th iterate, after $10 \tau$, almost coincides with the initial point. We have plotted 50 iterates, so we have 10 clusters of five points, showing that the rotation number (the ratio of the frequencies $\omega_{Q} / \omega_{f}$ ) is very close to $1 / 10$. This justifies the selection of $\omega_{Q}=5.035$ from the FFT.

Figure 14(a) shows the power spectral density for $M R W$ at $(R e, S t)=(615,50)$. The second frequency is $\omega_{Q}=3.996$, quite different and smaller than the frequency of $M S W$. In this case, we are even closer to resonance, but a different one. From the Poincare section in figure 13(b), we see that the iterates undergo two turns on the section before almost coinciding with the initial point after 25 iterates, so now the rotation number (frequency ratio) is $\omega_{Q} / \omega_{f}=0.07992 \approx 2 / 25$, and a very small frequency appears, $2 \omega_{f}-25 \omega_{Q} \approx 0.1$. Here, we can also estimate $\omega_{Q}$ by measuring the angle $\theta_{0}$ rotated by the flow pattern after one forcing period $\tau$, and the result is in full agreement with the Poincare section method.

The frequencies of $M R W$ and $M S W$ for $(R e, S t)=(615,50)$ are different, and so we have explored $\omega_{Q}$ as a function of $R e$, for fixed $S t=50$; the results are shown in figure 15. We have also plotted the critical frequency at the bifurcation point, $\omega_{Q, \mathrm{c}}=5.1572$, and the value of $\omega_{Q}$ for the most dangerous eigenfunction as a function of $R e$. What we observe is that the second frequency for the eigenfunction is almost constant, as is that for $M S W$ with $\omega_{Q}$ slightly smaller than the critical frequency $\omega_{Q, \mathrm{c}}$. In contrast, the second frequency of $M R W$ is substantially smaller than the critical value, and it decreases with $R e$, the amplitude of the forcing. This is probably related to the fact that the energy of the $M R W$ is much larger than the energy of $M S W$, and also to the larger subcriticality of the modulated rotating waves, as shown in figure 12 .

We see from figure 15 that $\omega_{Q}$ for $M R W$ and for $M S W$ at $(R e, S t)=(615,50)$ is very close to 4 and 5 , respectively. As the forcing frequency is $S t=50$, the ratio $\omega_{Q} / \omega_{f}$ is very close to rational $(2 / 25$ and $1 / 10$, respectively), as already noted from 


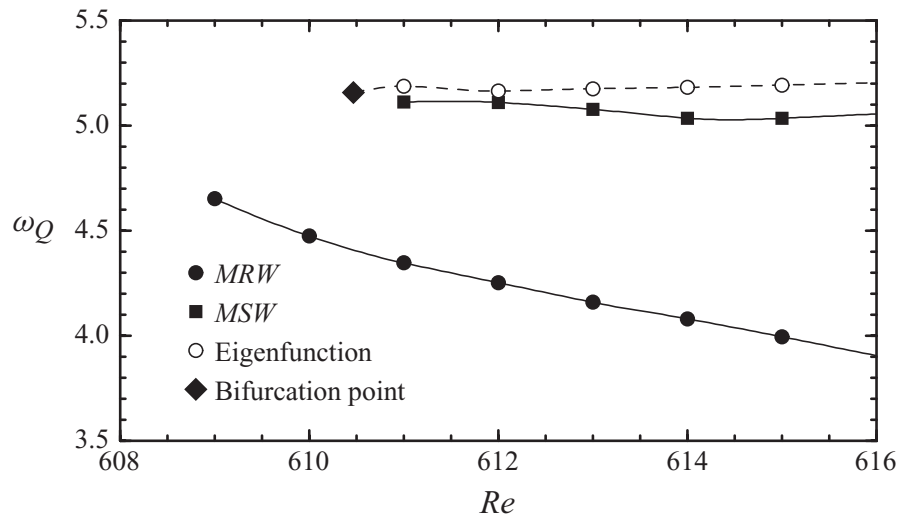

Figure 15. Computed frequencies $\omega_{Q}$ of the quasi-periodic states $M R W$ and $M S W$ as a function of $R e$. Also included are the frequencies of the most unstable eigenfunction, and the critical value

measuring the frequencies via FFT and Poincare sections. Of course, along the curves $\omega_{Q}(R e)$, other resonances can be located, but they all have large denominators, so no new dynamics associated with these resonances is expected (Kuznetsov 2004).

\section{Conclusions}

Several fluid systems with complete symmetry group $Z_{2} \times O(2)$ have been explored in recent years. The interest in these flows was triggered by the analysis of symmetric bluff body wakes (see a summary in Blackburn et al. 2005), starting with circular cylinders, and followed by other symmetric bodies, the square cylinder and a flat plate. Flows driven by the periodic motion of one of the container walls resulted in systems with the same symmetry group, and previous studies have analysed the rectangular-driven cavity (Blackburn \& Lopez 2003b; Leung et al. 2005) and a driven annular cavity (Blackburn \& Lopez 2011). Since the symmetry group of all these flows is the same, dynamical systems analysis (Marques et al. 2004) predicts the type of bifurcations they can undergo, which are the same in all cases regardless of the specifics of the problem and of the physical mechanisms at work. There are only three possibilities for the transition from the basic state to three-dimensional flows: synchronous modes preserving or breaking the space-time symmetry $H$ or quasi-periodic modes, that come in two flavours, either modulated travelling waves or modulated standing waves. The synchronous modes with the corresponding symmetry properties have been observed in all of these various flow problems. The quasi-periodic modes, however, have been much more elusive. In symmetric bluff-body wake problems, the quasi-periodic modes do not manifest themselves as primary bifurcations, and can only be observed or computed as secondary or higher bifurcations, in the form of mixed modes. In periodically driven flows, as there are more control parameters, they have been observed in the rectangulardriven cavity, but unfortunately spanwise endwalls effects, effectively breaking the $O(2)$ symmetry, resulted in quasi-periodic states that are neither spanwise symmetric nor spanwise travelling. In the annular-driven geometry with large radius ratios, the quasi-periodic modes have very large azimuthal wavenumbers and have not been found as nonlinearly saturated pure modes, but instead they are mixed complicated modes. 
In the present study, we have analysed the simplest geometry available with the correct symmetries, the cylindrical-driven cavity with moderate aspect ratio. This geometry has two advantages over previous studies. First, the $O(2)$ symmetry is exactly fulfilled by the cylindrical geometry (periodicity in the azimuthal direction), eliminating the spanwise endwall effects of the rectangular cavity. And second, the bifurcated states have small azimuthal wavenumbers (typically $m=1$ or 2 ), so the competition between different modes is greatly reduced. As a result, we have been able to compute nonlinearly saturated pure modulated standing and travelling waves for the first time, and we have also found the two types of synchronous modes, in the appropriate parameter ranges.

As a starting point of the analysis, the periodic synchronous base states have been computed for different forcing amplitudes, Re, and forcing frequencies, St. These are non-trivial states, with axisymmetric rollers forming alternately close to each of the endwalls due to the periodic oscillation of the cylinder sidewall. This oscillation produces axisymmetric jets of azimuthal vorticity, emerging from the corners, moving into the interior and forming the rollers.

The linear stability analysis of the base state has resulted in the computation of the marginal stability curve, shown in figure 3 . We have found synchronous bifurcations preserving the $H$ symmetry for small forcing frequencies $S t$, and breaking $H$ for larger $S t$ values. In between, for intermediate $S t$ values, we have found a transition to quasi-periodic solutions. The form of the instabilities is the same for all cases, in the formation of braids that are small-scale meridional perturbations of the rollers. The size and persistence of these braids depends strongly on St. These results are in good agreement with previous results in flows with the same symmetries, in particular with the driven rectangular cavity problem.

We have also computed and analysed in detail the saturated nonlinear states. In all cases, sufficiently close to the bifurcation curve, these are pure modes. The quasiperiodic stable solutions in the present problem are modulated rotating waves, and by restricting the computations to the appropriate subspace, we have also been able to compute the corresponding unstable modulated standing waves. As a result of these nonlinear simulations, we have established that the bifurcations to synchronous states are supercritical, while the bifurcations to quasi-periodic states are subcritical. A careful analysis of the quasi-periodic frequency $\omega_{Q}$ of the modulated standing waves has shown that $\omega_{Q}$ is almost constant and close to the frequency that emerges from the linear stability analysis for the modulated standing waves, whereas the modulated rotating waves exhibit a smaller frequency $\omega_{Q}$ that varies significantly with St. Finally, we have found in preliminary explorations, not yet reported, to higher $R e$ (i.e. increasing the forcing amplitude) that the three-dimensional pure modes undergo secondary bifurcations to complicated mixed modes for moderate increments in $R e$ beyond critical.

Future directions include an examination of the dynamics in the neighbourhood of the codimension-two points where two distinct modes bifurcate simultaneously; these codimension-two points act as organizing centres of the dynamics, and are very likely associated with the secondary bifurcations to mixed modes and more complex dynamics. We have found three codimension-two bifurcations, one associated with the competition between modes $B_{1}$ and $B_{2}$, and two on each side of the quasi-periodic region. The codimension-two bifurcation between $B_{1}$ and $B_{2}$ is a $1: 1$ resonancepreserving $H$-symmetry, that has been fully studied theoretically (Kuznetsov 2004). The other two bifurcations are more complex, they are bifurcations of maps with a real eigenvalue (either \pm 1 ) and a pair of complex conjugate eigenvalues of modulus 
1, where the $H$-symmetry plays a key role. Although there are some partial results on these bifurcations, they have not been yet fully analysed (Kuznetsov 2004), and here we have a physically realizable fluid dynamics system in which they occur. The normal form of the mode $\mathrm{A}$ and mode $\mathrm{B}$ interaction in a $Z_{2} \times O(2)$ spatio-temporal symmetric system, motivated by the cylinder wake flow, has been analysed by Barkley et al. (2000). Studies in fluids have been instrumental in developments in nonlinear dynamics, allowing for unified understanding of complex dynamics in a wide spectrum of fields. We hope that this driven cylinder system, which is relatively easy to conduct experiments in, can help further address some of these general open questions in dynamical systems.

Supplementary movies are available at journals.cambridge.org/FLM.

This work was supported by the National Science Foundation grants DMS05052705 and DMS-0922864, the Spanish Government grant FIS2009-08821 and the Korean Science and Engineering Foundation WCU grant R32-2009-000-20021-0.

\section{REFERENCES}

Allen, J. J. \& Lopez, J. M. 2007 Transition processes for junction vortex flow. J. Fluid Mech. 585, $457-467$.

Avila, M., Belisle, M. J., Lopez, J. M., Marques, F. \& Saric, W. S. 2008 Mode competition in modulated Taylor-Couette flow. J. Fluid Mech. 601, 381-406.

Avila, M., Marques, F., Lopez, J. M. \& Meseguer, A. 2007 Stability control and catastrophic transition in a forced Taylor-Couette system. J. Fluid Mech. 590, 471-496.

Barkley, D., Tuckerman, L. S. \& Golubitsky, M. 2000 Bifurcation theory for three-dimensional flow in the wake of a circular cylinder. Phys. Rev. E 61, 5247-5252.

Blackburn, H. M. \& Lopez, J. M. 2003a On three-dimensional quasiperiodic Floquet instabilities of two-dimensional bluff body wakes. Phys. Fluids 15, L57-L60.

Blackburn, H. M. \& Lopez, J. M. $2003 b$ The onset of three-dimensional standing and modulated travelling waves in a periodically driven cavity flow. J. Fluid Mech. 497, 289-317.

Blackburn, H. M. \& Lopez, J. M. 2011 Modulated waves in a periodically driven annular cavity. J. Fluid Mech. 667, 336-357.

Blackburn, H. M., Marques, F. \& Lopez, J. M. 2005 Symmetry breaking of two-dimensional time-periodic wakes. J. Fluid Mech. 522, 395-411.

Chossat, P. \& Iooss, G. 1994 The Couette-Taylor Problem. Springer.

Chossat, P. \& Lauterbach, R. 2000 Methods in Equivariant Bifurcations and Dynamical Systems. World Scientific.

Crawford, J. D. \& KNobloch, E. 1991 Symmetry and symmetry-breaking bifurcations in fluid dynamics. Annu. Rev. Fluid Mech. 23, 341-387.

Cross, M. C. \& Hohenberg, P. C. 1993 Pattern formation outside of equilibrium. Rev. Mod. Phys. 65, 851-1112.

Do, Y., Lopez, J. M. \& Marques, F. 2010 Optimal harmonic response in a confined Bödewadt boundary layer flow. Phys. Rev. E 82, 036301.

Golubitsky, M. \& Schaeffer, D. G. 1985 Singularities and Groups in Bifurcation Theory, vol. I. Springer.

Golubitsky, M. \& Stewart, I. 2002 The Symmetry Perspective: From Equilibrium to Chaos in Phase Space and Physical Space. Birkhäuser.

Golubitsky, M., Stewart, I. \& Schaeffer, D. G. 1988 Singularities and Groups in Bifurcation Theory, vol. II. Springer.

Hoyle, R. 2006 Pattern Formation, An Introduction to Methods. Cambridge University Press.

Iooss, G. \& Adelmeyer, M. 1998 Topics in Bifurcation Theory and Applications, 2nd edn. World Scientific.

Kuznetsov, Y. A. 2004 Elements of Applied Bifurcation Theory, 3rd edn. Springer.

LAMB, J. S. W. 1996 Local bifurcations in $k$-symmetric dynamical systems. Nonlinearity 9, 537-557. 
Lamb, J. S. W. \& Melbourne, I. 1999 Bifurcation from discrete rotating waves. Arch. Rat. Mech. Anal. 149, 229-270.

Leung, J. J. F., Hirsa, A. H., Blackburn, H. M., Marques, F. \& Lopez, J. M. 2005 Threedimensional modes in a periodically driven elongated cavity. Phys. Rev. E 71, 026305.

Lopez, J. M. \& HirsA, A. 2001 Oscillatory driven cavity with an air/water interface and an insoluble monolayer: Surface viscosity effects. J. Colloid Interface Sci. 242, 1-5.

Lopez, J. M. \& MARQues, F. 2000 Dynamics of 3-tori in a periodically forced Navier-Stokes flow. Phys. Rev. Lett. 85, 972-975.

Lopez, J. M. \& Marques, F. 2009 Centrifugal effects in rotating convection: nonlinear dynamics. J. Fluid Mech. 628, 269-297.

Lopez, J. M., Marques, F., Mercader, I. \& Batiste, O. 2007 Onset of convection in a moderate aspect-ratio rotating cylinder: Eckhaus-Benjamin-Feir instability. J. Fluid Mech. 590, 187-208.

Lopez, J. M., Marques, F., Rubio, A. M. \& Avila, M. 2009 Crossflow instability of finite Bödewadt flows: transients and spiral waves. Phys. Fluids 21, 114107.

Lopez, J. M. \& SHEN, J. 1998 An efficient spectral-projection method for the Navier-Stokes equations in cylindrical geometries I. Axisymmetric cases. J. Comput. Phys. 139, 308-326.

Marques, F. \& Lopez, J. M. 1997 Taylor-Couette flow with axial oscillations of the inner cylinder: Floquet analysis of the basic flow. J. Fluid Mech. 348, 153-175.

Marques, F. \& Lopez, J. M. 2000 Spatial and temporal resonances in a periodically forced extended system. Physica D 136, 340-352.

Marques, F., Lopez, J. M. \& Blackburn, H. M. 2004 Bifurcations in systems with $Z_{2}$ spatiotemporal and $O(2)$ spatial symmetry. Physica D 189, 247-276.

Marques, F., Mercader, I., Batiste, O. \& Lopez, J. M. 2007 Centrifugal effects in rotating convection: axisymmetric states and three-dimensional instabilities. J. Fluid Mech. 580, 303318.

Mercader, I., Batiste, O. \& Alonso, A. 2010 An efficient spectral code for incompressible flows in cylindrical geometries. Comput. Fluids 39, 215-224.

Mercader, I., Net, M. \& Falqués, A. 1991 Spectral methods for high order equations. Comput. Meth. Appl. Mech. Engng 91, 1245-1251.

Rubio, A., Lopez, J. M. \& Marques, F. 2010 Onset of Küppers-Lortz-like dynamics in finite rotating thermal convection. J. Fluid Mech. 644, 337-357.

Schlichting, H. \& Kestin, J. 1979 Boundary-Layer Theory, 7th edn. McGraw-Hill.

Vogel, M. J., Hirsa, A. H. \& Lopez, J. M. 2003 Spatio-temporal dynamics of a periodically driven cavity flow. J. Fluid Mech. 478, 197-226. 